\title{
Response to letter
}

We greatly appreciate Drs Gholamrezanezhad, Mirpour and Jazayeri's insightful comments regarding our article: " $85 \%$ of maximal age-predicted heart rate is not a valid exercise endpoint". ${ }^{1}$ The authors of the letter discuss a number of valid issues to be considered when performing physical stress testing. Essentially, they raise the question: "If $85 \%$ of maximal heart rate is not an acceptable exercise end point, what is?"

The main purpose of exercise stress testing in patients suspected of having coronary artery disease is to assess whether symptoms are reproduced by increasing cardiac metabolic demand through graded physical exercise. Accordingly, symptoms (i.e. ischemic chest pain, shortness of breath or severe fatigue) must be endpoints of exercise testing.

The authors of the letter are correct in stating that each of these presumably ischemic symptoms may occur before $85 \%$ of maximal age-predicted heart rate is achieved and that in fact ischemia may be the very cause for the inability to increase heart rate further. We agree with the authors that when unequivocal ischemia is provoked, the exercise test is a valid diagnostic test. However, in our article ${ }^{1}$ we challenge the practice of stopping exercise testing at an arbitrary target heart rate, before limiting symptoms occur.

Of note, all patients in our study who did not achieve target heart rate, stopped because of limiting symptoms at a mean achieved workload of $9.1+3.5$ metabolic equivalents (METs), without a difference between patients on or not on beta-blockers. Almost one-half of these patients achieved a workload exceeding 10 METs. This group had a high prevalence of inducible ischemia on myocardial perfusion imaging. It should be recognized that they represent a small and selected group because most patients with perceived low exercise capacity were switched beforehand to pharmacological stress.

Particularly at low workload (i.e. $\leq$ stage 2 Bruce protocol, $<7$ METs), it may be difficult to determine with certainty if a patient is limited by inducible ischemia or by deconditioning or lack of motivation. Unless one is unequivocally convinced that the patient developed myocardial ischemia, it would be prudent to switch to pharmacological stress rather than repeat the same

J Nucl Cardiol 2012;19:632.

$1071-3581 / \$ 34.00$

Copyright (c) 2012 American Society of Nuclear Cardiology. test at the same low level of exercise with imaging. If the result of imaging test were to be negative, one is still in the same conundrum because of low workload.

Myocardial ischemia is induced by increased demand or workload and not by increased heart rate alone. Accordingly, we always mention in our exercise reports the total number of METs achieved at peak exercise. The relationship between METs and exercise stages was validated in Bruce's original work ${ }^{2}$ and has been used in many subsequent studies addressing the relationship between exercise performance and outcome. We do agree with the authors' suggestion that the 'double product' (peak exercise heart rate $\times$ systolic blood pressure) may also be an acceptable alternative to estimate cardiac workload. ${ }^{3}$

Thus, the diagnostic validity of physical exercise testing is first and foremost determined by the ability to reproduce symptoms that were the reason for testing. However, in practice many patients do not reproduce their symptoms. Under the latter circumstances, exercise should be continued, as Dr Bruce urged, to the patient's limit. $^{2}$ Whereas peak exercise heart rate does not constitute a valid exercise endpoint, exercise-induced limiting symptoms are valid reasons to terminate exercise, regardless of heart rate achieved.

Frans J. Th. Wackers, MD

Mohit Jain, MD

Cardiology, Yale University, New Haven, CT frans.wackers@yale.edu

\section{References}

1. Jain M, Nkonde C, Lin BA, Walker A, Wackers FJ. $85 \%$ of maximal age-predicted heart rate is not a valid endpoint for exercise testing. J Nucl Cardiol 2011;18:1026-35.

2. Bruce RA, Blackmon JR, Jones JW, Strait G. Exercise testing in adult normal subjects and cardiac patients. Pediatrics 1963;32: 742-56.

3. Sadrzadeh Rafie AH, Sungar GW, Dewey FE, Hadley D, Myers J, Froelicher VF. Prognostic value of double product reserve. Eur J Cardiovasc Prev Rehab 2008;15:541-7.

doi:10.1007/s12350-012-9537-6 